\title{
Myeloma cells in the cerebrospinal fluid in plasma cell neoplasia
}

\author{
A. M. AFIFI ${ }^{1}$ \\ From Cairo, Egypt
}

SYNOPSIS Myeloma cells were detected in the cerebrospinal fluid of two patients with plasma cell neoplasia during the myelographic studies of 38 patients whose myeloma was associated with $\vec{\circ}$ extensive neurological complications. The myeloma cells were looked for in Wright stained centrifuged deposit of 2-5 ml samples of the cerebrospinal fluid obtained during myelography. The possi- $\vec{\omega}$ bility that occult traumatic lumbar puncture had allowed entry of circulating myeloma cells from the peripheral blood into the subarachnoid space was excluded by the absence of myeloma cells in smears of peripheral blood and its buffy coat. Up to the end stages of the disease the meningeal $\omega$ myeloma lesions remained microscopical and no signs of raised intracranial tension were manifested by either patient.

Neurological complications in generalized myelomatosis are mostly in the form of cord or root compression by extradural myeloma or vertebral collapse secondary to skeletal lesions. Other less common complications are amyloid infiltration of peripheral nerves and various demylinating non-metastatic complications of systemic malignancy. Compared with acute lymphoblastic leukaemia, where central nervous system deposits develop in about $50 \%$ of patients, intradural cerebral or spinal myeloma lesions developing during the course of systemic myelomatosis are only rarely encountered in the myeloma patients submitted to careful clinical and necropsy studies. Only one patient with meningeal myeloma complicating generalized multiple myelomatosis (Maldonado et al., 1971) and five patients with isolated intracranial myeloma were reported by different authors (Clark, 1954; Medoc et al., 1961 ; Weiner et al., 1966; Moosy et al., 1967; Gravanis et al., 1969). This paper reports two patients with plasma cell neoplasia whose cerebrospinal fluid was proved by cytological study to contain myeloma cells.

1 Address for reprints: Dr A. M. Afifi, 9B Abou El Feda St, Zamalek Cairo, Egypt.

\section{CASE 1}

A 48 year old man was admitted to hospital January 1969 with severe anaemia, progressive loss weight, and pain in the left clavicle. Physical examirte tion revealed severe pallor, mild oedema of the low limbs, no lymphadenopathy, and no hepatospleno-

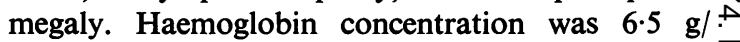
$100 \mathrm{ml}$, leucocyte count $4,600 / \mathrm{mm}^{3}$, with $60 \%$ neutrophils, platelets $195,000 / \mathrm{mm}^{3}$, and erythrocytic sedimentation rate $100 \mathrm{~mm} /$ hour. Bone marrow aspirate was hypoplastic and heavily infiltrated with $\stackrel{2}{2}$ myeloma cells which formed $45 \%$ of the nucleated $\stackrel{\unrhd}{\complement}$ elements of the aspirate. Total serum proteins were $\overrightarrow{\vec{O}}$ $8.8 \mathrm{~g} / 100 \mathrm{ml}$ and serum protein electrophoresis 3 revealed a myeloma band at the $\beta$-position. Skeletal survey revealed generalized osteoporosis of the spine, ribs, and pelvic bones, and an extensive osteolytic lesion of the left clavicle causing almost complete destruction of the middle third. Bone biopsy from: the destroyed area of the left clavicle showed complete destruction of the normal bone structure, which was replaced by sheets of myeloma cells with few macrophages and small areas of haemorrhage. Threeo months from diagnosis of the disease, the patient developed bilateral progressive wasting of the uppero and lower limbs with almost equal involvement of the proximal and distal muscle groups. Both fundios were normal. Pneumoencephalography failed to $N$ show any intracranial space occupying lesion and N myelography proved the absence of spinal block. 


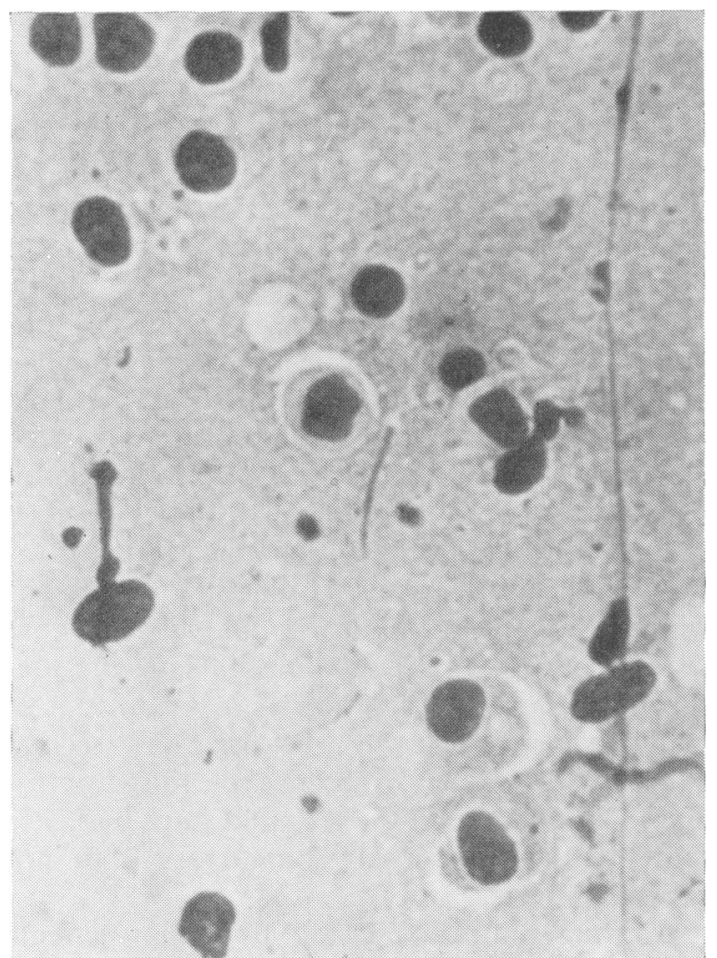

FIG. 1. Case 1. Myeloma cells in the CSF. Wrightstained film.

Cerebrospinal fluid specimens obtained during lumbar puncture revealed the presence of myeloma cells in small quantities (Fig. 1). Serum oxaloacetic transaminase level was 56 units/l. (normal, up to 20) serum pyruvic transaminase 46 units (normal, to 15 ), serum aldolase 68 units/l. (normal, to 33). Deltoid muscle biopsy showed marked variation in the diameter of the muscle fibres with focal areas of necrosis and perivascular cellular infiltration by lymphocytes, plasma cells, and neutrophils.

In the ensuing few months the patient developed weakness of the muscles of deglutition and respiration followed by fatal bronchopneumonia. Up to the end stages of the disease no myeloma cells could be detected either in the peripheral blood films or in the buffy coat films.

At necropsy the cranial dura mater looked normal and could be stripped without difficulty from the overlying bone. The skull bones were not infiltrated by any tumours. Section of the right cerebral hemisphere revealed scattered subarachnoid infiltrates of myeloma cells in the form of sheets occupying the

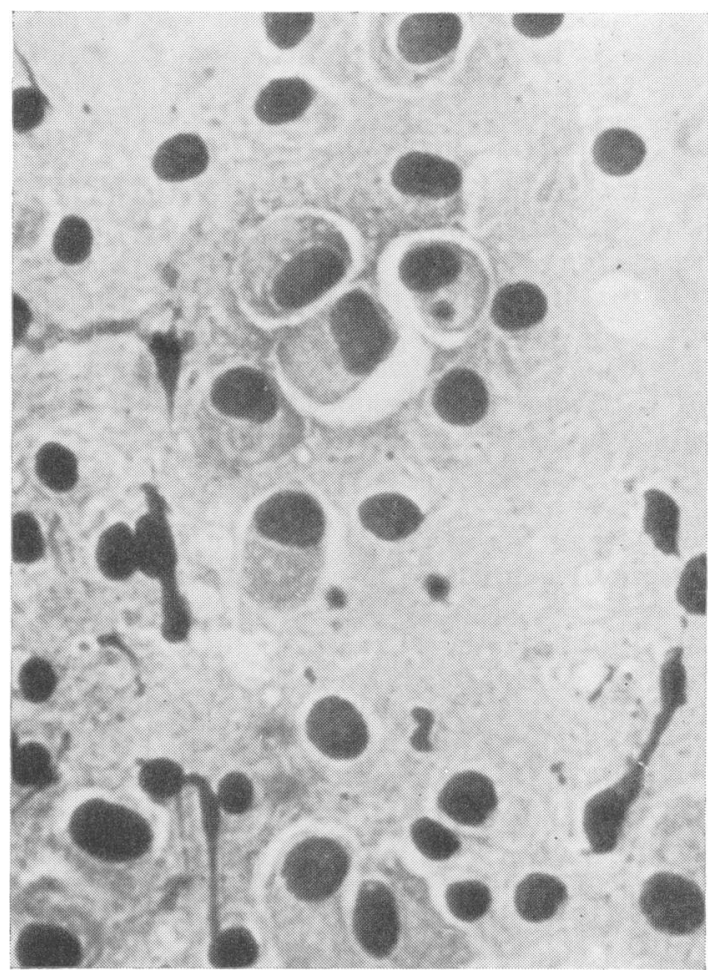

FIG. 2. Case 2. Myeloma cells in the CSF. Wrightstained film.

subarachnoid space of the precentral and postcentral sulci. No myeloma deposits could be detected in other areas of the central nervous system. The liver, lymph nodes, kidneys, and lungs did not show macroscopic myeloma lesions. The spleen was invaded by two small nodules which were formed of fairly well differentiated plasma cells.

\section{CASE 2}

A 45 year old man presented with bilateral weakness and wasting of the hand muscles together with weakness in extending the wrists. In three months he developed bilateral wrist drop, and difficulty in walking, with unsteady gait. Neurological examination revealed symmetrical wasting of the thenar, hypothenar, and interosseous muscles of both hands together with marked wasting of the extensor muscles of the wrist and only slight wasting of the wrist flexors. Lower limb examination revealed impairment of vibratory and position sensation in both legs, diminution of the ankle jerks, but normal 
knee jerks. There was no weakness or wasting of the muscles of the lower limbs. Haemoglobin concentration was $10 \mathrm{~g} / 100 \mathrm{ml}$, leucocyte count $8,000 / \mathrm{mm}^{3}$ with normal differential, platelets $75,000 / \mathrm{mm}^{3}$ and sedimentation rate $123 \mathrm{~mm} / \mathrm{hr}$. Chest radiograph was clear and barium meal for the stomach and duodenum revealed normal findings. There was no hepatosplenomegaly or lymphodenopathy.

In the ensuing few months the patient developed excruciating back pain in the lower dorsal region with spastic weakness of the lower limbs and retention of urine. Neurological examination showed spastic paraplegia. Myelography revealed complete thecal block extending from the ninth to the eleventh dorsal vertebra. At operation an extradural greyish fleshy mass constricting the spinal cord both posteriorly and laterally was found extending from the upper border of the ninth dorsal vertebral body to the lower border of the eleventh. The overlying dura mater was intact but there were vascular adhesions between it and the tumour. After successful surgical removal, histological study showed sheets of myeloma cells intermingled with a minor population of eosinophil and neutrophil cells.

Cytological examination of cerebrospinal fluid obtained during myelography revealed the presence of myeloma cells in the Wright stained smears of the centrifuged deposit (Fig. 2). Sternal marrow aspirate showed normal cellularity with heavy infiltration of the smears by myeloma cells, which formed over $50 \%$ of the nucleated elements of the aspirate. Serum protein electrophoresis showed a dense monoclonal band midway between the beta and gamma regions.

The patient was started on intermittent melphalan therapy to which he showed partial response. Over the 18 months of his follow-up, repeated examination of the peripheral blood and buffy coat smears failed to detect any myeloma cells. The patient eventually died of uncontrollable attacks of haematemesis and melaena due to persistent thrombocytopaenia.

\section{DISCUSSION}

Although the number of cases of meningeal myeloma described in the literature is too small to draw valid conclusion about its pathogenesis and clinical course, the available clinical evidence suggests that it differs from meningeal leukaemia in many aspects. In addition to its rarity, which contrasts with the high incidence of meningeal leukaemia, the occurrence of isolated meningeal myeloma with exclusive involvement of the central nervous system is a feature which has not been reported in meningeal leukaemia. The absence of myeloma cells in the blood of our patients excludes the possibility of development of meningeal myeloma as a result of invasion of the central nervous system by circulating neoplastic cells, a mechanism postulated by West $e t \stackrel{\text { ? }}{=}$ al. (1972) as responsible for the development of $\underset{\overrightarrow{2}}{\overrightarrow{0}}$ meningeal leukaemia. Meningeal myeloma is presumed to develop as a result of neoplastic $\underset{\vec{\rho}}{\overrightarrow{\vec{S}}}$ proliferation into myeloma cells of the toti- $\overline{0}$ potent mesenchymal cells accompanying the pial 흘 blood vessels. This mechanism may be held $\frac{\bar{\omega}}{T}$ responsible for the development of both isolated $\stackrel{\Phi}{\varnothing}$ meningeal myeloma and meningeal lesions com- œ plicating systemic myelomatosis. A possible, $\vec{\circ}$ though rarely reported, mechanism is the inva- $\overrightarrow{-}$ sion of the dura mater by epidural myelomas $\vec{\omega}$ which seed myeloma cells in the subarachnoid space. These two suggested mechanisms are presumed to be responsible for the development of $\omega$ meningeal myeloma in our first and second $\vec{\circ}$ patients respectively. Absence of raised intra․ cranial pressure until the terminal stage indicate that the intracranial myeloma mass does nof 0 expand rapidly.

Although not manifested by other patients with meningeal myeloma, the microscopge nature of the meningeal lesions in our patient demonstrates another feature not reported meningeal leukaemia. This finding can be $\mathrm{ex}-\overrightarrow{0}$ plained by the fact that myelomas with widespread lymphoreticular extension into the liver, spleen, and most lymph node groups have high proliferative activity and produce tumour masses in the nervous system as described in the patient of Maldonado et al. (1970), while myelomas largely contained within the bone marrow have low proliferative activity and tend to involve the nervous system, as demonstrated in the present two cases, by microscopic lesions that do not show any progress to the tumour stage till the end stages of the disease.

\section{REFERENCES}

Clarke, E. (1954). Cranial and intracranial myelomas. Brain, $\bigcirc$ 77, 61-81.

Gravanis, M. B., Someren, A. (1969). Intracranial solitary $\frac{D}{O}$ plasmacytoma. (Abstract.) American Journal of Clinical Pathology, 52, 769.

Maldonado, J. E., Kyle, R. A., Ludwig, J., and Okazaki, H. (1970). Meningeal myeloma. Archives of Internal Medicine, 126, 660-663.

Medoc, J., Rodriguez, B., and Rodríguez, Juanotena, J. (1961). 
Mieloma meningeo. Anales de la Facultad de Medicina Montevideo, 46, 82-91.

Moossy, J., and Wilson, C. B. (1967). Solitary intracranial plasmacytoma. Archives of Neurology, 16, 212-216.

Weiner, L. P., Anderson, P. N., and Allen, J. C. (1966).
Cerebral plasmacytoma with myeloma protein in the cerebrospinal fluid. Neurology (Minneap.), 16, 615-618.

West, R. J., Graham-Pole, J., Hardisty, R. M., and Pike, M. C. (1972). Factors in pathogenesis of central-nervoussystem leukaemia. British Medical Journal, 3, 311-314. 